\section{$\underset{\substack{\text { hommes } \\ \text { \& migrations }}}{ }$}

\section{Hommes \& migrations}

Revue française de référence sur les dynamiques

migratoires

1302 | 2013

Le Japon, pays d'immigration?

\title{
Amin Maalouf, Les Désorientés
}

Paris, Grasset, 2012, 520 p., $22 €$.

\section{Mustapha Harzoune}

\section{Q OpenEdition \\ 1 Journals}

\section{Édition électronique}

URL : http://journals.openedition.org/hommesmigrations/2506

DOI : 10.4000/hommesmigrations.2506

ISSN : 2262-3353

\section{Éditeur}

Musée national de l'histoire de l'immigration

\section{Édition imprimée}

Date de publication : 1 avril 2013

Pagination : 191-193

ISBN : 978-2-919040-22-3

ISSN : 1142-852X

\section{Référence électronique}

Mustapha Harzoune, «Amin Maalouf, Les Désorientés », Hommes \& migrations [En ligne], 1302 | 2013, mis en ligne le 12 septembre 2013, consulté le 22 septembre 2020. URL : http://

journals.openedition.org/hommesmigrations/2506 ; DOI : https://doi.org/10.4000/ hommesmigrations.2506 


\section{LIVRES}
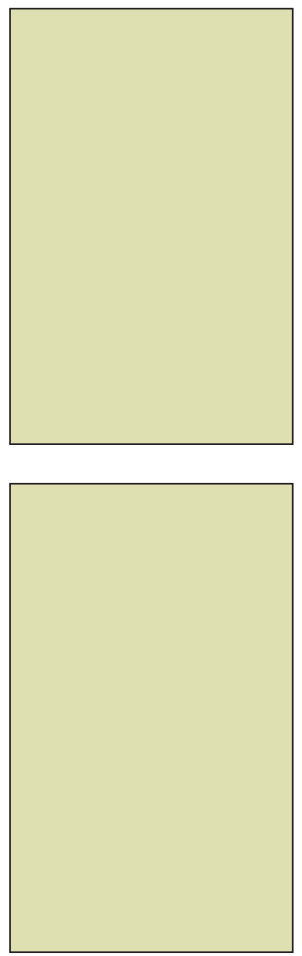

\section{Yu Miri}

Le Berceau

au bord de l'eau

Arles, éd. Philippe Picquier, coll. "Japon”, 2000, 224 p., traduit par Jean Campignon

\section{Yan Sogiru}

Sang et os

Monaco, éd. du Rocher, coll. "Série japonaise",

2011, 560 p.,

traduit par R. Sekiguchi

et P. Honnoré

Parmi les auteurs coréens ou d'origine coréenne vivant au Japon et s'exprimant en japonais, deux ont été traduits en français. Le Berceau au bord de l'eau est le roman le plus autobiographique de Yu Miri, qu'elle publia en 1997 alors âgée de 29 ans. Yan Sogiru avait, lui, 62 ans lorsqu'il sortit son roman Sang et os inspiré de l'histoire de sa famille au Japon. Sang et os a été adapté à l'écran par Sai Yôichi en 2004 avec Takeshi Kitano dans le rôle principal.

Ils sont tous les deux Coréens du Japon de deuxième génération. L'une a grandi à Yokohama et l'autre à Osaka, deux villes qui abritent encore aujourd'hui d'importantes communautés coréennes. Le roman de Yu Miri est un récit introspectif au fil de souvenirs d'enfance dans une famille dont elle cherche à recoller les différentes versions des raisons de l'immigration dans les années d'après-guerre : idéologie politique, petits trafics, dissensions familiales, grossesse hors mariage. Le passage plus ou moins clandestin au Japon de ses grands-parents maternels et de son père illustre le caractère souvent complexe du départ. Le Berceau au bord de l'eau est aussi, comme beaucoup de ses autres œuvres, une réflexion sur l'identité des Coréens du Japon.

De même, dans Sang et os, les personnages ont fait le voyage entre la Corée et le Japon à l'époque coloniale pour des raisons très diverses : mariages malheureux, discriminations internes à la Corée, pauvreté. Très différent de l'ouvrage intimiste de Yu Miri, le roman de Yan Sogiru est une fresque d'un demisiècle de la communauté coréenne à Osaka. Centré sur la figure d'un personnage brutal, Kim Junpyeong, l'auteur fait se croiser les vies de femmes coréennes à la fois entreprenantes et soumises aux normes traditionnelles du mariage, d'activistes coréens, mais aussi d'enfants coréens ou métis de la deuxième génération. Cette épopée familiale aborde la question du travail - exploitation des Coréens à l'époque coloniale et entreprenariat ethnique aux lendemains de la guerre -, celle de l'engagement politique - depuis les premières mobilisations collectives contre les discriminations coloniales jusqu'à la promotion des "retours" en Corée du Nord dans les années 1960. Elle propose le regard d'une minorité nationale sur les grands temps de l'histoire, telles la dépression économique des années 1930, la guerre du Pacifique et la décolonisation.

Dans les deux ouvrages, la question du nom se pose, emblématique à la fois du regard porté par les gouvernants sur 
la population coréenne et des hésitations des Coréens entre assimilation et revendication de leur identité. Dans le roman de Yan Sogiru, les personnages utilisent entre eux leur nom coréen, mais les Japonais les appellent selon la prononciation japonaise des caractères de leur nom. Dans la dernière période de la colonisation, le Japon décida dans le cadre de sa politique d'assimilation d'attribuer à chacun un nom de famille japonais afin d'affirmer formellement son appartenance à l'empire. Ainsi, le personnage principal du roman, Kim Junpyeong, devient Kanamoto Shunpei. Pendant longtemps, après la guerre, la naturalisation japonaise supposait l'adoption d'un nom japonais. Même non naturalisés, un certain nombre de Coréens continuent d'utiliser un nom japonais dans la vie courante afin d'éviter les discriminations.

Le nom est souvent le dernier élément qui permet de distinguer les Coréens

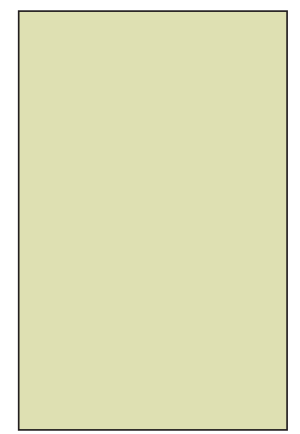

\section{Mathias Enard}

Rue des voleurs

Paris, Actes Sud, 2012,

252 p., 21,50€

Dès le début, j’ai été pris par ce livre: Mathias Enard a un style, qui s'impose dès les toutes premières lignes. C'est rare. Ensuite, on peut ouvrir le roman à n'importe quelle page : tout de suite, la voix revient, caractéristique, inimitable. C'est un rythme particulier, avec une phrase parfois très de deuxième ou troisième génération et, face aux discriminations, beaucoup ont cherché à rester invisibles tout en essayant de maintenir au sein du foyer leur culture d'origine. Le récit de Yu Miri s'ouvre précisément par une analyse de son nom. Son grand-père, qui décida de son prénom, a choisi des caractères qui se prononçaient de la même façon en japonais et en coréen. Par chance, le caractère de son nom de famille pouvait aussi passer pour un nom de famille japonais: "Supposons que j'aie eu un nom hurlant le Coréen, comme Kim quelque chose, par exemple, il est probable que le cours de ma vie aurait été fort différent de ce qu'il est actuellement." Pourtant, à ses débuts dans le théâtre, entre la prononciation coréenne, Yu, et la japonaise, Yanagi, elle opte pour Yu, et choisit donc que ses origines coréennes soient visibles dans la société japonaise.

Hélène Le Bail

longue et pourtant jamais pompeuse ou verbeuse. C'est une phrase en déplacement permanent, une phrase migrante, prenant appui sur une extraordinaire mécanique des points-virgules (Enard est un orfèvre du point-virgule, dans toutes ses nuances, dans toute son amplitude), et qui peut épouser aussi bien les sinuosités de la réflexion intérieure que la description d'un paysage de Barcelone ou de Tunis.

C'est aussi un livre courageux et intelligent. Courageux par son sujet, sujet brûlant qui aurait pu mener aux pires 
banalités ou, à l'inverse, aux pires extrémités (sur les printemps arabes, l'attentat de Marrakech, la tuerie de Toulouse, l'islam, le terrorisme). Intelligent par le choix des sites et des personnages, ainsi que par le traitement qu'il leur réserve : le choix de Tanger, par exemple, villefrontière qui nous rappelle à chaque rue que le destin du monde arabe est le nôtre, et en même temps ville en marge, tout comme le beau personnage de Lakhdar, le narrateur, complexe et séduisant, narrateur-chien fou, tour à tour irritant, amusant, émouvant. Roman plein de poésie également, pas de cette "poésie" qui consiste à enrober les phrases avec de beaux adjectifs bien cousus et rutilants, mais d'une poésie pour ainsi dire interne, naissant de la syntaxe et du rythme autant que du vocabulaire et des images. La première phrase, par exemple, est époustouflante. Enfin, c'est un livre loin des clichés, loin de l'ignorance phénomé- nale et de la bêtise qui s'empare des bouches et se pose sur les micros quand on aborde ces sujets. C'est un livre qui maîtrise parfaitement son projet, avec un humour à la fois féroce et fragile, un livre transpercé aussi par l'ironie et l'insolence, le tout servi par une langue superbe. Il faut le dire : par son sujet, par son style, par son ton, Rue des voleurs n'est pas seulement un bon ouvrage de plus sur l'exil, mais un grand livre qui sait nous arracher à nos certitudes, à nos indifférences ou à nos ignorances, pour nous faire entendre une voix singulière prise dans le tumulte du monde. C'est un livre fragile, parfois hilarant, souvent émouvant. Un livre vivant: "La vie est une machine à arracher l'être; elle nous dépouille, depuis l'enfance, pour nous repeupler en nous plongeant dans un bain de contacts, de voix, de messages qui nous modifient à l'infini, nous sommes en mouvement."

Michaël Ferrier

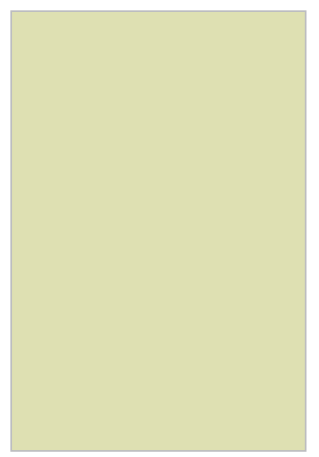

\section{Alain Mabanckou}

Lumières

de Pointe-Noire

Paris, Seuil, 2013,

304 p., $19,50 €$

Lumières de Pointe-Noire nous conte un retourau pays natal, traversé de vertiges et d'une émotion d'autant plus forte qu'elle est contenue. Intelligemment, le livre s'ouvre sur une légende congolaise énigmatique : nous entrons d'emblée dans un monde différent, relié à la force mystérieuse des origines ainsi qu'à leur puissance parfois délétère. Un monde qu'on ne peut renier mais dont il faut savoir s'extraire. Par instants, l'histoire tâtonne un peu, suivant en quelque sorte une démarche en crabe, de celles que le narrateur décrit lui-même dans une très belle page: "On croit qu'ils vont aller à gauche, ils font demi-tour, ils s'arrêtent sans savoir pourquoi, ils tournent en rond, et ils repartent en vitesse vers la droite avant de revenir à gauche. Mais ce que j'aime chez les crabes c'est qu'ils savent 
toujours où ils vont aller, et ils finissent par arriver tôt ou tard." Car, sous son allure de pèlerinage parfois chaotique, c'est un livre bien construit, scandé par des chapitres qui portent tous des titres de classiques du cinéma : le narrateur multiplie ainsi les travellings et les zooms sur une ville qu'il voit à travers le filtre de ses souvenirs, avec un zeste d'amertume. II a sans doute aussi besoin de cet écran, à la fois pour retrouver cette ville et cette période de sa vie, mais aussi pour s'en détacher.

Rencontres, voix, lumières, ambiances, retrouvailles quelquefois drôles, d'autres fois désenchantées, surmontées de l'ombre absente de la mère, qui ouvre le livre, et de celle de grand-mère Hélène, qui le clôt.

Régulièrement, des photographies viennent ponctuer le texte, comme si l'écriture renonçait à fouiller plus avant, comme si la réflexion s'arrêtait pour céder la place à un album de souvenirs couleur sépia, dont l'auteur ne sait finalement plus trop quoi faire. Alors s'ouvre un silence; une hésitation se glisse entre les points de suspension et les photographies : impuissant, le texte laisse la place à ces images simples, grignotées par le temps, "accablées par la poussière des regrets". Et d'une certaine manière, cela rend aussi ce livre très attachant. Michaël Ferrier

\section{Amin Maalouf \\ Les Désorientés \\ Paris, Grasset, 2012, 520 p., $22 €$}

Les Désorientés d'Amin Maalouf dressent le tableau des désillusions d'une génération. Celle de ces hommes et femmes qui avaient 20 ans dans les années 1970 et se réveillent des décennies plus tard, hébétés. Le thème rappelle L'Orient après l'amour (Actes Sud, 2008) de Mohamed Kacimi, dans lequel le Franco-Algérien constate l'écart entre une jeunesse algérienne qui en 1975, reprenant le "ni Dieu ni Maître" de Léo Ferré à Sidi-Ferruch, scandait "la Rabi-ou-la Nabi" ("Ni Dieu ni Prophète"), et cette autre jeunesse qui, dix ans plus tard, s'en va, kamisisée et niqabellisée, inquiéter les consciences, enlaidir les rues d'Alger et vo(i)ler son soleil. "Notre utopie est derrière nous. Nous étions tellement convaincus de vivre rive gauche que nous n'avons rien vu venir", constate Kacimi.

Maalouf écrit à partir de son expérience, de l'histoire d'un autre pays, le Liban, jamais cité, avec aussi sa sensibilité et cette humanité qui font de lui un auteur et un être précieux. Pour démonter les mécanismes qui vont faire passer le monde, à tout le moins une génération, du "rêve" à "l'enfer", il met en scène Adam, un Franco-Libanais exilé à Paris qui s'en revient, pour la première fois, dans la ville où il est né et a grandi. II tentera de reconstruire, pour un soir, le cercle bigarré des amis d'enfance aujourd'hui éparpillés et divisés par la guerre-qui, quelque vingt-cinq ans plus 
tôt, se retrouvaient dans la grande maison de Mourad pour refaire le monde. Adam le laïc sera l'artisan de ces retrouvailles qui devraient réunir la belle et indépendante Sémiramis, Tania la veuve de Mourad, Nidal devenu islamiste, Ramzi, converti en frère Bazile et retiré dans un monastère, Ramez, riche ingénieur installé avec sa famille à Amman, Naïm le juif parti à Sao Paulo et Albert Kithar qui a fui aux États-Unis la guerre et l'opprobre, pour vivre en paix son homosexualité.

Le récit progresse entre le journal tenu par Adam, quelques photos et courriers conservés, les échanges de mails et les dialogues nombreux. II faut oser deux bémols : l'amalgame entre les différents registres ne prend pas. Cette fragilité de même que des dialogues souvent pâlots et ampoulés laissent entendre le côté factice de la construction. Autre bémol, la liaison entre Sémiramis et Adam placée sous la surprenante bénédiction de Dolorès, la compagne restée à Paris. Tout cela paraît un peu emberlificoté mais permet à Maalouf d'écrire sur le couple, l'amour, et d'assener: “C'est d'abord en nous ligotant le corps que les tyrannies morales nous ligotent l'esprit", qui résonne comme un écho au "mon corps m'appartient et il est l'honneur de personne" de la Tunisienne Amina.

Pourtant, ces 520 pages retiennent l'attention. D'abord par l'intrigue, dont le dénouement n'intervient qu'à la toute fin du récit. Ensuite par l'intelligence (et l'urgence) du propos sur des thèmes chers à l'auteur des Identités meurtrières:l'omniprésence envahissante des religions, les fragmentations communautaires et identitaires, la civilisation arabe perçue comme une "civilisation vaincue", déconnectée de la marche du monde, le mépris occidental, la liberté individuelle, celle des corps et des cœurs. À ces thèmes, le Franco-Libanais en ajoute au moins un : le retour au pays de l'exilé.

Les Désorientés s'ouvrent sur un coup de fil : Tania avertit Adam que Mourad est en train de mourir et qu'il souhaite revoir son vieil ami une dernière fois. Adam, qui vit depuis plus de vingt ans à Paris, hésite. Il a depuis longtemps jugé et condamné Mourad pour ce qu'il a fait pendant la guerre. Lui, à la différence de Mourad, refuse la logique des enfermements et la guerre des appartenances, "je suis né sur une planète, pas dans un pays", dit-il.

Mourad est resté au Liban quand Adam est parti,cequia fait del'un "un coupable" et de l'autre "un exilé". Tania, veuve fidèle et vindicative, lui sert le refrain connu de la "trahison" et de la bonne conscience à bon compte : "La question n'est pas de savoir ce que tu aurais fait si tu étais resté. La question est de savoir ce que serait devenu le pays si tout le monde était parti, comme toi. Nous aurions tous gardé les mains propres, mais à Paris (...)." Ici, la plume de Maalouf - "Tout homme a le droit de partir, c'est son pays qui doit le persuader de rester." - rappelle celle du Chinois Ha Jin, immigré aux ÉtatsUnis: "La Chine m'a trahi, alors je refuse de rester plus longtemps son sujet" (La Liberté de vivre, Seuil, 2011). Et si Adam n'est jamais retourné au Liban, ce n'est pas par nostalgie : "De la disparition du passé, on se console finalement, c'est de la disparition de l'avenir qu'on ne se remet pas." 
Celui qui est devenu un émigré, incertain et hésitant quant à ses comportements, restera seize jours, seize jours d'un "pèlerinage inutile" vers le passé, le temps de la jeunesse et des illusions. Le prénom Adam n'est pas ici le signe d'une humanité naissante, plutôt celui d'une humanité qui s'éteint. "Le mémorialisteest pour les siens un traitre, ou tout au moins un fossoyeur. Tous les mots affectueux qui viennent sous ma plume sont des baisers de mort." Comme l'écrit Camus dans Caligula, "on ne revient pas en arrière et il faut aller jusqu'à la consommation". Qu'importe ! Adam va travailler à reformer, le temps d'une soirée, ce cercle des amis disparus, ce cercle où "aucun d'entre nous ne ressemblait à sa communauté". Il y a urgence, le pays, la planète même sont en sursis. Et si cette mission symbolique qu'il s'est assignée semble condamnée par avance, il n'entend pas, lui, renoncer: "Et quand viendra mon tour, je tomberai comme un tronc, sans avoir plié, et en répétant à qui voudra l'entendre : 'C'est moi qui ai raison et c'est l'Histoire qui a tort!"' Mustapha Harzoune

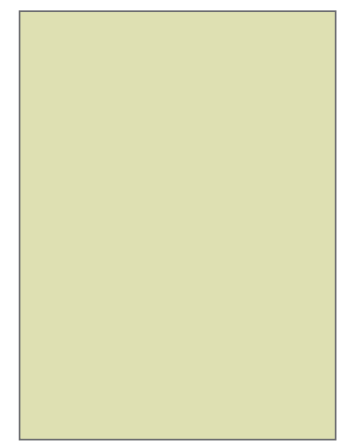

Katrina Kalda

L’Arithmétique des dieux

Paris, Gallimard, 2012, 224 P., 16,90€

L'Arithmétique des dieux fait partie de ces livres qui, dès la première phrase inspirent confiance. II en est des livres comme des êtres, un regard, un mot, la petite musique du style ou du comportement, et vous voilà d'entrée conquis... ou méfiant.

L'Arithmétique des dieux, c'est un ton, une langue, pudique, feutrée, élégante et surtout sincère. Pas d'esbroufe ou de nombrilisme, de contentement de soi dans ce roman qui mêle l'Histoire - la domination soviétique et la déportation des Estoniens au lendemain de la guerre - et l'introspection de la narratrice. Arrivée en France avec sa mère à l'âge de l'enfance, Kadri, devenue profes- seure d'université, "lêtre le plus rationnel de la famille", bataille, pourtant et encore, avec "les esprits de l'eau et des bois" de son Estonie natale. Pour elle, "il est devenu patent que la branche de la famille dont je suis issue possède un talent rare pour rater sa vie".

Katrina Kalda raconte cette histoire en alternant le récit de Kadri avec les lettres de Lisbeth, adressées dans les années 1940 à Elda, sa grand-mère. Kadri est insomniaque, en proie à des cauchemars, des angoisses, des poussées de "fièvre de rangement". Bourrée aux somnifères, elle cultive une "incompétence sentimentale", des "blessures d'amour-propre", du "ressentiment", en un mot Kadri sombre dans une dépression profonde.

En 1940, "l'hiver des grandes déportations", Lisbeth fut expédiée pour vingt ans dans un goulag sibérien. Comme tant d'autres, elle appartenait à une famille classée ennemie de l'État, 
entendre d'indépendantistes estoniens. Le roman avance au rythme des courriers de Lisbeth, des journées et des souvenirs de Kadri. Le lecteur, par touches délicates, par circonvolutions pudiques, pénètre l'intimité de la famille Raud tout entière dominée par la despotique grand-mère Elda : fidèle du régime communiste, directrice d'une usine, l'État lui avait accordé un logement dans lequel logeaient trois générations de Raud. Kersti, la mère de Kadri, pour qui "prendre un époux équivalait à s'enterrer vivante", a fini par détester toute la smala. En 1989, munie de deux visas touristiques, elle embarque avec sa fille pour un aller simple en direction de Paris, laissant derrière elle l'Estonie encore soviétique, l'autoritaire bellemère, Juhan, son époux maniaque du rangement qui bibine à la vodka et fume comme un pompier. Paris, "la ville de tous les possibles", allait sûrement tendre ses bras à cette femme, francophile, professeur de piano, à la carrière "fichue" par les Raud et une trop précoce maternité. II n'en sera rien et, comme un
Driss Chraïbi débarqué de son Maroc natal, elle découvre que la Ville Lumière rime aussi avec misère noire.

Les souvenirs, ceux de l'enfance, des vacances à Tallinn, du quotidien gris et oppressant de l'Estonie soviétique, l'histoire de la guerre et de la déportation, jusqu'aux secrets de famille enfermés à double tour pour éviter d'éveiller la suspicion des autorités, finissent par incarner ces "êtres maigres et sinueux (...) marchant ou rampant sur le paysage. (...) Exsangues, ressemblant plus à des branches mortes qu'à des humains", comme ceux peints par oncle Oskar. L'exil n'empêchera pas les blessures de continuer à suppurer. Pour se libérer, délivrer les vivants et épargner ceux à venir "de ce je-ne-sais-quoi qui empoisonnait les corps, fermait les visages et empêchait de parler", l'anamnèse débouchera sur une autre exigence : il faut accepter de ne pas tout savoir de cette "arithmétique des dieux". Comme les scilles de Sibérie, les premières fleurs à sortir après l'hiver sibérien, Kadri pourra alors renaître à la vie. $M$. $H$. 


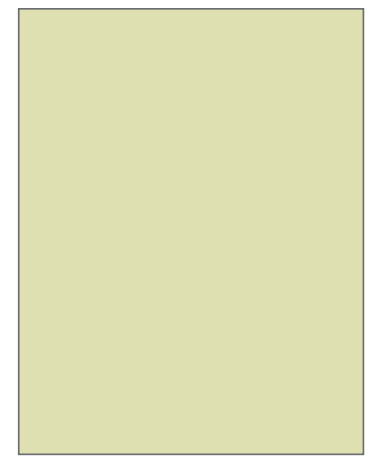

\section{Frédéric Ciriez}

\section{Mélo}

Paris, Verticales, 2013 325 p., $20 €$

Un syndicaliste suicidaire,un immigrécongolais conducteur de benne à ordures et roi de la sape, une Française d'origine chinoise championne de la vente au détail sur les trottoirs de la capitale. Trois personnages et trois récits pour une unité de lieu : Paris, symbole de ces villes-mondes, kaléidoscopiques, bigarrées, ethnicisées, individualisées, espace de l'intersection et des bifurcations, de l'ombre et de la lumière. La ville se montre ici à travers les vitres d'une Xantia, du haut d'un camion benne ou à la vitesse des rollers qui filent de rue en rue. Il y a le Paris côté jour et le Paris de la nuit ; le Paris illuminé des Grands Boulevards et celui sale et malodorant des ordures ; le Paris de la "rue du faubourg Saint-Denis, la rue du monde entier" et celui d'une histoire oubliée au 14, rue de Paradis; le Paris cosmopolite des enseignes et des commerces, des populations et des quartiers, le Paris des juifs, Kabyles, Russes, Africains, Turcs, Chinois, Bangladais et celui du populo, de la resquille, des toutous à sa mémère et de la flicaille en civil, de la prostitution version africaine ou version asiatique, le Paris "hype" et celui des bouis-bouis ; le Paris des humanités séparées, celui des mannequins anorexiques et celui d'un syndicaliste romantique et dépressif.

Unité de lieu et unité de temps. En cette veille de $1^{\text {er }}$ Mai, un syndicaliste lorientais décide d'en finir quand "Parfait de
Paris", maître es-sapologie, prince de l'élégance, se prépare pour son heure de gloire à la grande soirée de "la lutte à mort des paraître". Moment grandiose, éblouissant, de défis vestimentaires et de joutes oratoires, sur une bande-son tout en rumbas. Au même moment, la jeune Barbara Xiao tient les comptes de son commerce et de ses désillusions amoureuses.

Trois titres enfin pour trois récits : "Transfixion", "Transformation" et "Transaction" qui scandent autant d'identités. Une, anonyme, marquée par le souci de l'autre et la quête de liberté devenue socialement invisible. L'autre, cachée derrière un surnom et qui fait dire: "Quinze ans que je suis en France et c'est ça le résultat. Je ne suis qu'un émigré esthétique, incompris du petit peuple blanc dégénéré." La dernière, insaisissable, métissée de Barbara Xiao. Des identités qui se retrouvent dans les trajectoires urbaines des trois protagonistes : périphérique, à contresens et en contresens ou tout en zigzag.

Ce qui relie ces personnages ? Des SMS, un briquet, une ou deux rues à Paris et à Saint-Ouen, un après-midi au cinéma. Autant dire pas grand-chose.

Frédéric Ciriez déploie un sens du détail et de l'observation, une précision chirurgicale portant aussi bien sur les qualités techniques d'une moto, les critères de l'élégance façon sapeur ou l'industrie des déchets urbains. Le style, affûté, se déploie à partir d'un travail d'orfèvre, particulièrement documenté, où la langue elle-même devient un objet traduisant la marche du monde II lui suffit de quelques phrases, quelques images, des faits et des lieux pour incarner trois 
individualités. L'écriture cinématographique progresse à coups de projecteur, de flashs, de retours en arrière, de plans larges. Pas de discours sur l'immigration ou l'intégration, pas d'approche psychologisante ou sociologique, du concret, rien que du concret : un couteau de boucher, une Dunhill, une fragrance d'Antaeus de Chanel, une plongée dans le Triangle d'or d'Aubervilliers, un briquet... et se dessine le visage d'une société. Mélo est un roman taoïste où les vides disent les pleins, où les connexions et les entailles du monde passent par un jeu de miroir où se reflètent autant de convergences que de bifurcations. C'est ce qui fait que l'écriture de Ciriez est une écriture poétique, qui libère l'imaginaire et agite les neurones.

M. H.

\section{Sylvie Weil}

Le Hareng

et le Saxophone

Paris, Buchet Chastel, 2013, 496 p., 23

Sylvie Weil est d'une famille et d'un continent pour qui I'histoire compte. Aussi, quand elle débarque, du jour au lendemain, dans la famille Shackman installée depuis des décennies à Brooklyn, le décalage est patent. Nous sommes en terre américaine, "terre d'amnésie", d'amnésie "sélective", et dans une famille qui ne retient du passé que quelques moments choisis. Sylvie, la "frenchie" en baguenaude à New York, rencontre Eric Weitzner. Après seulement deux rendez-vous, ils décident de se marier "à la sauvette", mariage civil expédié en sept minutes. Cela ne sera pas du goût de Molly Weitzner née Shackman, mère d'Eric. Molly ne va pas se gêner pour le faire comprendre à sa belle-fille, balayant du même coup "l'admirable pedigree" des Weil. "Je sens que mes relations avec ma belle-mère vont être intéressantes", écrit Sylvie avec une distance amusée. La "réfugiée" de France, comme l'appellent les membres du centre culturel juif du Bronx où s'est installéle couple, découvre ses nouveaux voisins, des rescapés des camps nazis ou de vieilles dames qui, assises sur des chaises pliantes alignées le long des trottoirs du quartier, forment une sorte de comité central de vigies, bienveillantes et bavardes.

L'auteure, qui se demande "dans quelle famille suis-je entrée", va se plonger dans l'histoire des Shackman. Elle va revisiter les lieux des origines, Ouman en Ukraine, et remonter six générations en arrière, jusqu'à l'ancêtre fondateur de la lignée, un orphelin de 9 ans joueur d'échecs, qui de ce fait choisit Shackman pour nom de famille.

Sylvie Weil croise l'histoire collective des juifs ukrainiens, celle des réfugiés débarqués aux États-Unis et la saga familiale. Si la première est connue, la seconde, qui s'ouvre aux alentours de 1810 et se poursuit un siècle plus tard avec l'arrivée du premier Shackman à Brooklyn, renferme quelques particularités, histoires et secrets de famille 
où se détachent les figures des arrièregrands-parents Shmiel-Haïm et Esther, et des grands-parents, Guédalia et Rivka ou encore de Clara, devenue la yiddishe mame de l'auteure et la mémoire du clan.

Le commerce du hareng permit à trois générations de Shackman, si ce n'est de faire fortune, à tout le moins de prospérer. Quant au saxophone, il appartenait à Sam, le beau-père et le mari effacé de Molly. Un saxophone qu'il a dû vendre en 1929, crise oblige. Un saxophone dont il dut faire le deuil sa vie durant, Molly oblige.

L'enquête est présentée sans aucune pesanteur. Sylvie Weil alterne I'histoire lointaine et les épisodes de l'exil des Shackman. Par petites touches, comme en grappillant dans les souvenirs et les rares documents, elle reconstitue la vie de la famille depuis l'Ukraine jusqu'aux dernières générations, dont certains

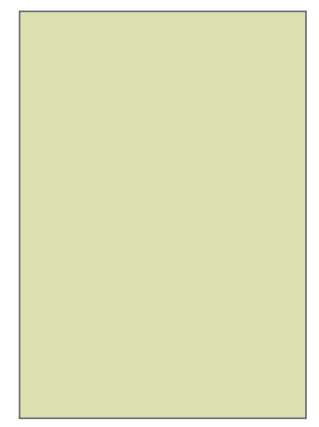

Yassaman Montazami Le Meilleur des jours Paris, Sabine Wespieser, 2012, 138 p., 15

Le Meilleur des jours est un livre lumineux à l'image de son personnage central : Behrouz, exilé iranien à Paris, éternel étudiant et père de la narratrice. Une lumière qui se retrouve dans le style de Yassaman Montazami : léger, drôle, vif, attendrissant.

Le fils de Rosa fut un prématuré, condamné à rejoindre le sort des autres membres vont "se dissoudre dans le melting pot" nord- américain au grand dam des anciens. Plusieurs fois, elle montre que ces Shackman constituent "une puissante machine à oublier", que "dans cette famille on ne pleurniche pas, on ne s'attendrit pas, à peine si on s'embrase". Alors, "Pourquoi parler de la mort ?" Pourquoi ressasser quand on est en Amérique et qu'ici les "circonstances" obligent à aller de l'avant, obligent à réussir, quitte à faire ce que plusieurs pogroms, une guerre et une révolution en Europe n'ont pas réussi à faire : violer le shabbat en ouvrant le samedi et proposer, en les tenant tout de même bien à l'écart des traditionnels filets de harengs, pickels et autre langue fumée, du jambon et du bacon! "Après s'être transporté, il faut se transformer." Une page de l'immigration juive aux ÉtatsUnis écrite avec humour et tendresse. M. H.

enfants mort-nés. Jusqu'à ce jour où, poussant enfin un cri, il s'éveille à la vie et inonde de bonheur le cœur de sa mère. En souvenir de cette journée exceptionnelle, elle le prénomme "Beyrouz", "le meilleur des jours" en persan. Behrouz, en révolutionnaire digne de ce nom et grâce à maman Rosa, célébrissime et richissime auteure de livres de cuisine, ne travailla pas de sa vie entière, occupé qu'il était à la rédaction de sa thèse consacrée à Marx. Cette thèse, pour laquelle il s'exila en France à la fin des années 1960, fut la grande affaire de sa vie, pourtant elle ne vit jamais le jour. 
Insatiable de connaissances, l'homme, toujours, remit sur le métier son œuvre qui en devint virtuelle, à jamais. Personne ne connaitra donc "la cause originaire de l'inégalité entre les hommes" et le monde ne deviendra pas "meilleur". Qu'importe, Beyrouz a laissé davantage que des milliers de feuillets noircis en farsi ou les 3 ooo livres de sa bibliothèque. l'homme fut un être généreux, facétieux, hospitalier - pour l'opposition de gauche comme pour une aristocrate nostalgique du Shah--, un homme respectueux de tous, à commencer par sa fille, qu'il considéra non comme une enfant mais comme "un sujet de plein droit doué d'un libre arbitre". Murmurant à l'occasion des vers de Hâfez: "Bois, car qui a vu comment s'achève l'œuvre du monde, s'est délivré prestement du chagrin et a pris la lourde coupe", il fut plus poète que militant.

Les trente années de mariage avec Zahra, la "lunaire", furent simplement affectueuses. L'amour attendait, ailleurs. À Téhéran. II s'appelait Bibi, Bibi la "solaire", qui au temps lointain de la faculté, après l'annonce des fiançailles de Beyrouz et Zahra, "se jeta dans le mariage comme on se lancerait dans le vide par une fenêtre".
Avec Darius, Bibi et Beyrouz formeront un ménage à trois. La fantaisie de Beyrouz réussit à distraire l'époux frappé de neurasthénie depuis la Révolution islamique. Ensemble ils fument du haschish, ensemble ils conversent, ensemble ils lisent et commentent les poèmes de Hâfez, ensemble ils fredonnent et dansent sur des vieux tubes de Boney M. Comme chez Amin Maalouf (Les Désorientés), le retour de l'exilé annonce les retrouvailles avec les anciens camarades, les rescapés de "l'armée des ombres". Ici, la culpabilité domine : face à Bijan, "brisé" par cinq années d'emprisonnement et de tortures, "Beyrouz fut pris d'une gêne incommensurable. II ne savait que lui dire, il n'osait le regarder. (...) Mon père se sentait soudain coupable de ne pas avoir été embastillé lui aussi. (...) II aurait voulu fuir."

Le Meilleur des jours est une miniature littéraire dédiée à un homme rare et attachant, une sorte de saint laïc, à michemin entre un personnage d'Albert Cossery et un bouddha rieur, n'était le physique, sans doute, de cet amateur de whisky et de canard à l'orange cuisiné les nuits d'insomnie et offert en guise de petit déjeuner à sa fille.

M. H 